\title{
Optimization Procedure for Wideband Matched Feed Design
}

\author{
Palvig, Michael Forum; Jørgensen, Erik; Meincke, Peter; Breinbjerg, Olav
}

Published in:

Proceedings of 2016 10th European Conference on Antennas and Propagation

Link to article, DOI:

10.1109/EuCAP.2016.7481136

Publication date:

2016

Document Version

Peer reviewed version

Link back to DTU Orbit

Citation $(A P A)$ :

Palvig, M. F., Jørgensen, E., Meincke, P., \& Breinbjerg, O. (2016). Optimization Procedure for Wideband Matched Feed Design. In Proceedings of 2016 10th European Conference on Antennas and Propagation IEEE. https://doi.org/10.1109/EuCAP.2016.7481136

\section{General rights}

Copyright and moral rights for the publications made accessible in the public portal are retained by the authors and/or other copyright owners and it is a condition of accessing publications that users recognise and abide by the legal requirements associated with these rights.

- Users may download and print one copy of any publication from the public portal for the purpose of private study or research.

- You may not further distribute the material or use it for any profit-making activity or commercial gain

- You may freely distribute the URL identifying the publication in the public portal 


\title{
Optimization Procedure for Wideband Matched Feed Design
}

\author{
Michael Forum Palvig ${ }^{1,2}$, Erik Jørgensen ${ }^{2}$, Peter Meincke ${ }^{2}$, Olav Breinbjerg ${ }^{1}$ \\ ${ }^{1}$ Department of Electrical Engineering, Electromagnetic Systems, Technical University of Denmark, Kgs. Lyngby, Denmark \\ ${ }^{2}$ TICRA, Copenhagen, Denmark
}

\begin{abstract}
The inherently high cross polarization of prime focus offset reflector antennas can be compensated by launching higher order modes in the feed horn. Traditionally, the bandwidth of such systems is in the order of a few percent. We present a novel design procedure where the entire matched feed and reflector system can be efficiently optimized. This allows the design parameters of the matched feed to be directly related to the desired design goals in the secondary pattern over a specified band. Using this procedure, we present a design of a die-castable axially corrugated matched feed horn that provides an XPD improvement better than $7 \mathrm{~dB}$ over a $12 \%$ bandwidth for a reflector with an $f / D$ of 0.5 . An investigation of the mode requirement for an arbitrary circular aperture feed is also presented.
\end{abstract}

Index Terms-matched feed, reflector antenna, horn antenna, optimization, antenna modelling.

\section{INTRODUCTION}

Offset parabolic reflector antennas suffer from high cross polarization compared to their symmetric counterparts. On the other hand, they are very attractive in terms of avoiding feed and strut blockage. The cross polarization arises from tilting the otherwise perfectly polarized feed antenna away from the paraboloid apex. A solution to this was proposed by Rudge and Adatia in 1975 [1]. The idea is to use a non-perfectly polarized feed that has exactly the cross polarization that will cancel the one introduced by the asymmetry of the antenna system.

Rudge and Adatia examine the antenna in receiving mode of a plane wave from boresight. The focal region field in this situation was described some years earlier by Bem [2]. They find that this field can be approximately matched by the addition of the $\mathrm{TE}_{21}$ mode in a circular feed, the $\mathrm{HE}_{21}$ in a radially corrugated feed, or the $\mathrm{TE}_{11}$ mode in a rectangular feed. Their practical implementation is a Potter horn [3] with a $\mathrm{TE}_{21}$ mode launcher consisting of two pins inserted into the waveguide. There are many variations in the literature with pyramidal horns [4], corrugated horns [5], and feed arrays [6]. Mode launchers are usually some variation of either a pin/post in the waveguide or a slot/stub, but other concepts like exciting the higher order mode in the outer region of a coaxial horn [7] or a series of non-concentric irises [8] have also been published.

The challenge for designing a matched feed system is the bandwidth. The mode generation and mode propagation along a waveguide are dispersive phenomena and thus, performance of these feeds quickly deteriorate when the frequency moves away from the design frequency. To improve this, the full desired frequency band must be included in the design optimizations.

\section{Required Modes For MATCHEd Condition}

As mentioned in the previous section, the fact that the required extra circular waveguide mode is $\mathrm{TE}_{21}$, is usually justified from the focal region fields when a plane wave is incident on the reflector [1] (described in more detail in [9]). The method gives a good indication of the solution to the problem, but is not strictly rigorous. In principle, the procedure should be repeated for all angles of incidence within the main beam, and how should these different focal region fields be combined when designing the feed? Also, the focal field will have ringing effects due to the abrupt truncation of the reflector aperture field which cannot be reproduced with the feed.

In this paper, we validate the use $\mathrm{TE}_{21}$ for matched feeds by a more rigorous procedure. We let a circular aperture with a combination of waveguide modes illuminate the offset reflector. The mode coefficients, aperture size, and aperture pointing is then optimized to yield a satisfactory field distribution in the reflector aperture. Given that the definition of a satisfactory aperture field is judiciously chosen, this method will yield a good overall antenna. The figure of merit is chosen as

$$
F=\eta_{A} \eta_{s}-K_{c r} \mathcal{E}_{c r},
$$

where $\eta_{A}$ and $\eta_{s}$ are the aperture and spillover efficiencies, respectively. $K_{c r}$ is a constant weighing the importance of the cross-polar aperture error, $\mathcal{E}_{c r}$, defined as

$$
\mathcal{E}_{c r}=\frac{\iint_{A}\left|E_{c r}\right|^{2} d A}{\iint_{A}\left(\left|E_{c o}\right|^{2}+\left|E_{c r}\right|^{2}\right) d A},
$$

with $A$ being the projected aperture of the reflector. Maximizing the aperture and spillover efficiencies results in high gain, and minimising $\mathcal{E}_{c r}$ will enforce uniform polarization in the aperture and thus low cross polarization in the far-field. For a good antenna, a compromise between these goals must be made. Here, we maximize $F$ for a chosen $K_{c r}=10$, which is found experimentally to be a good compromise. The parameters to the optimization are complex excitation coefficients to a spectrum of radiating waveguide modes, as well as the aperture radius and tilt angle. The radiated field 
from a given mode is computed using numerically power normalized formulas from [10].

We let the antenna be offset in the vertical direction. Fig. 1 shows the optimized mode amplitudes as a function of $f / D$ for the two polarizations. Superscripts denote the two degenerate modes for an $m, n$ pair, i.e. $\mathrm{TE}_{11}^{2}$ is a $90^{\circ}$ rotated version of $\mathrm{TE}_{11}^{1}$ and $\mathrm{TE}_{21}^{2}$ is a $45^{\circ}$ rotated version of $\mathrm{TE}_{21}^{1}$. The regular co-polar part of the feed signal is seen as a combination of the fundamental $\mathrm{TE}_{11}$ mode and the $\mathrm{TM}_{11}$ mode as in the Potter horn. The required cross-polar part can be obtained by inclusion of $\mathrm{TE}_{21}$, but can be improved by also including $\mathrm{TM}_{01}$ or $\mathrm{TE}_{01}$ for vertical and horizontal polarization, respectively. This fact is most often neglected. In fact, the $\mathrm{TE}_{21}$ mode can be left out, and $\mathrm{TE}_{01}$ and $\mathrm{TM}_{01}$ used instead. However, good results can also be obtained without the $m=0$ modes, but more $\mathrm{TE}_{21}$ is then needed. In the following we shall focus on designing a matched feed based on $\mathrm{TE}_{21}$ generation, but a future investigation may show that feeds with $\mathrm{TM}_{01}$ and $\mathrm{TE}_{01}$ are just as good or better.

The drawback of the mode optimization technique is that it is hard to control where the optimization finds a maximum, and the maximum may not be the global maximum. For this reason, the curves in Fig. 1 are not smooth. However, for all $f / D$ values, the plotted mode combination provides a satisfactory reflector aperture distribution with nearly parallel field lines.

\section{REFLECTOR-FEED OPTIMIZATION}

The GRASP software tool [11] offers highly accurate and efficient Physical Optics (PO) as well as Method of Moments (MoM) implementations. The latter is combined with the former in a domain decomposition technique [12] to jointly optimize feed-reflector systems. The interior of the feed itself can be split up into several devices. Generalized scattering matrices are computed for the waveguide ports of each device. Devices can then be cascaded to obtain a combined scattering matrix of the entire feed. Scattering matrices are computed using either 3D MoM, Body of Revolution (BoR) MoM, or Mode Matching techniques. The analysis method does not have to be the same for the different devices.

For radiation analysis, the interior scattering matrices of the horn are coupled to the exterior geometry using MoM. Rigorous coupling to the reflector is achieved through either PO or MoM/MLFMM. The final cascaded system can be used to calculate the field at any point given an arbitrary waveguide excitation.

When changing one variable of the antenna system, only the scattering matrices dependent on this variable need to be recomputed before cascading to obtain the entire antenna scattering matrix. This is very useful for optimization purposes and means that intermediary optimisation goals, like phase edge taper and beam width of the feed can be skipped, and the antenna system can be directly optimized based on the final far-field goals. By avoiding these indirect quantities, a better compromised design can be found over a larger bandwidth.
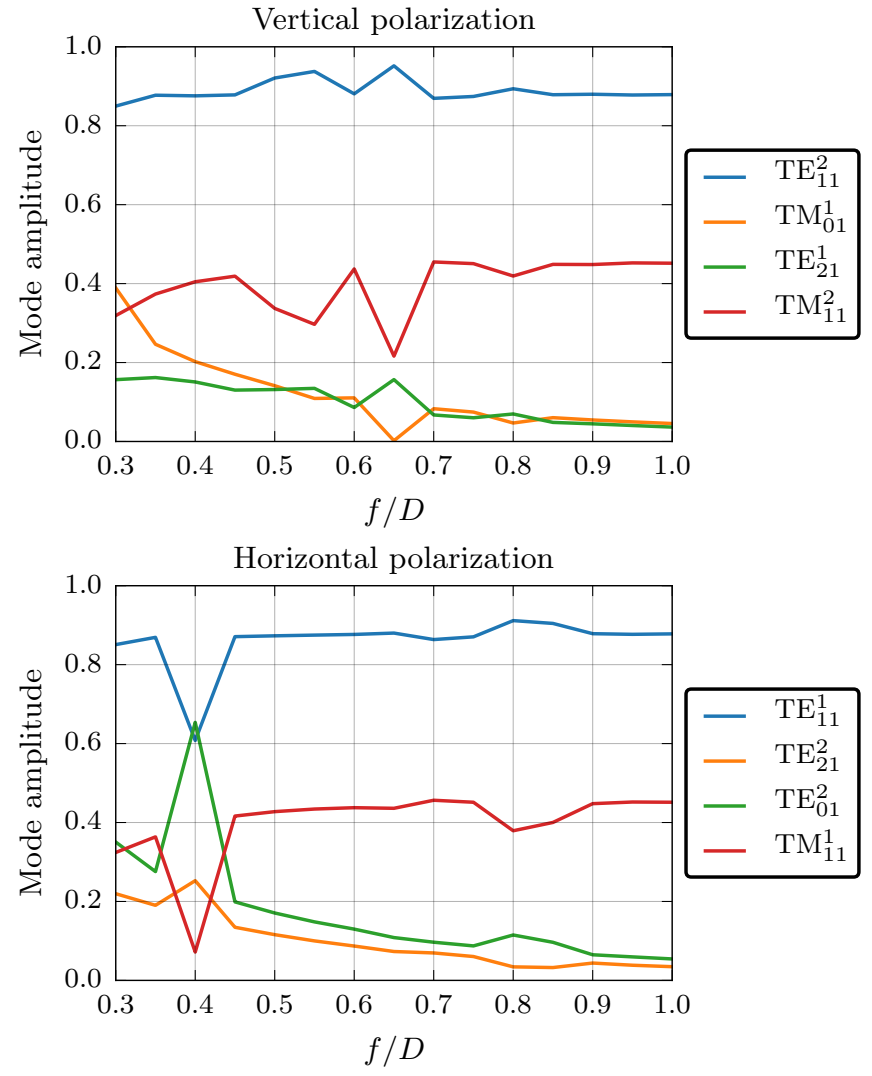

Fig. 1. Optimized mode content as a function of $f / D$. Superscripts enumerate the two degenerate field solutions for each mode type.

These properties have been used in the matched feed design described in the following section.

\section{Axially Corrugated Matched Feed}

Using the general mode guidelines of Section II and the tool described in Section III, a matched feed has been designed. The design is based on the axially corrugated horn. This feed type can be optimized to have good cross-polar performance over a reasonable bandwidth, and is much simpler to fabricate than the radially corrugated horn, as well as being shorter and lighter. The fact that the horn is short is also a desirable property when it comes to matched feeds or multimode feeds in general, because different modes have different phase velocities in the waveguide. The phase velocity of each mode is dependent on frequency and thus, the longer a waveguide or horn, the more sensitive the phase relationship between modes is to frequency.

The goal is to make a feed which reduces cross polarization for both linear polarizations in frequency band $27.5 \mathrm{GHz}$ to $31 \mathrm{GHz}$. This constitutes a relative bandwidth of $12 \%$ which is considerable in the context of matched feeds. The feed will illuminate an $f=0.25 \mathrm{~m}$ parabolic reflector with a $D=$ $0.5 \mathrm{~m}$ circular aperture diameter and an offset clearance of $D^{\prime}=0.05 \mathrm{~m}$.

The design will be carried out in the following four steps: 


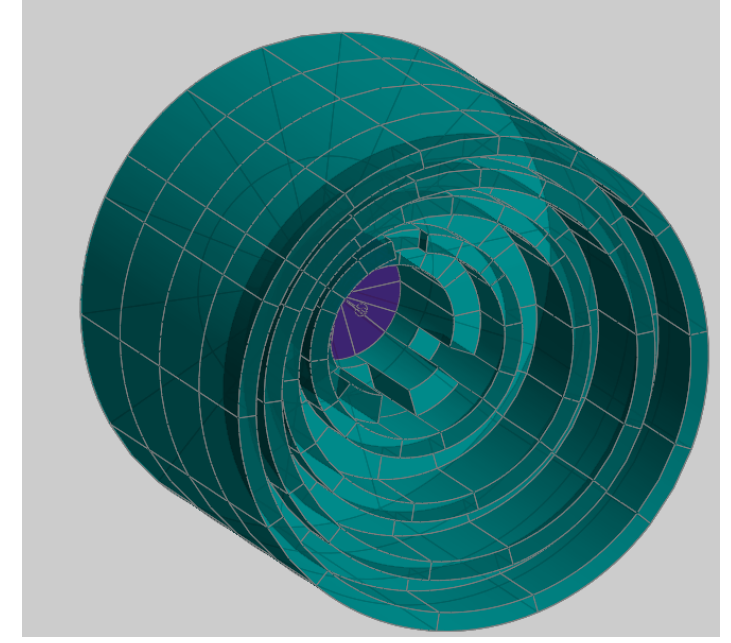

Fig. 2. Axially corrugated horn with modifications for matching. Radius of input waveguide is $3.5 \mathrm{~mm}$ and aperture radius is $12.3 \mathrm{~mm}$.

1) A standard axially corrugated horn is optimized in CHAMP [13] to illuminate the given reflector with low feed cross polarization over the band.

2) The feed is imported into GRASP. Mode converting elements are added and scattering parameters are optimized over the band to approximately generate the amount of $\mathrm{TE}_{21}$ given in Fig. 1 .

3) Mode converting elements are optimized, minimising the far-field maximum cross-polar level, while keeping the directivity intact.

4) All horn parameters are optimized.

\section{A. Mode Conversion}

In order to transfer some energy into the $\mathrm{TE}_{21}$ mode, a part of the geometry must be rotationally asymmetric. We have chosen to make asymmetries that preserve the ability to die-cast the horn. The chosen artefacts are cutouts, or chips, in the corrugation ridges as shown in Fig. 2. The innermost corrugation of the depicted horn has zero slot width/depth and thus collapses to a step. Likewise, the chips can be viewed as slots.

The middle chip or slot in each corrugation generates the proper $\mathrm{TE}_{21}$ from the horizontally polarized fundamental mode and the two slots on either side generate $\mathrm{TE}_{21}$ for the vertically polarized fundamental mode.

The principle of the horn and mode launcher is very similar to that of a Eutelsat patent [14].

\section{B. Results}

The cross-polar level for both linear polarizations is shown in Fig. 3 over the band. A cross-polar reduction better than $7 \mathrm{~dB}$ is achieved over the $12 \%$ frequency bandwidth. A graphical representation of the feed and reflector is shown in Fig. 4. Pattern cuts for both polarizations at one frequency are shown in Fig. 5. The cuts are in the horizontal plane where the cross-polar level is largest in this case (but not necessarily in general). There are still many variations to the mode launcher

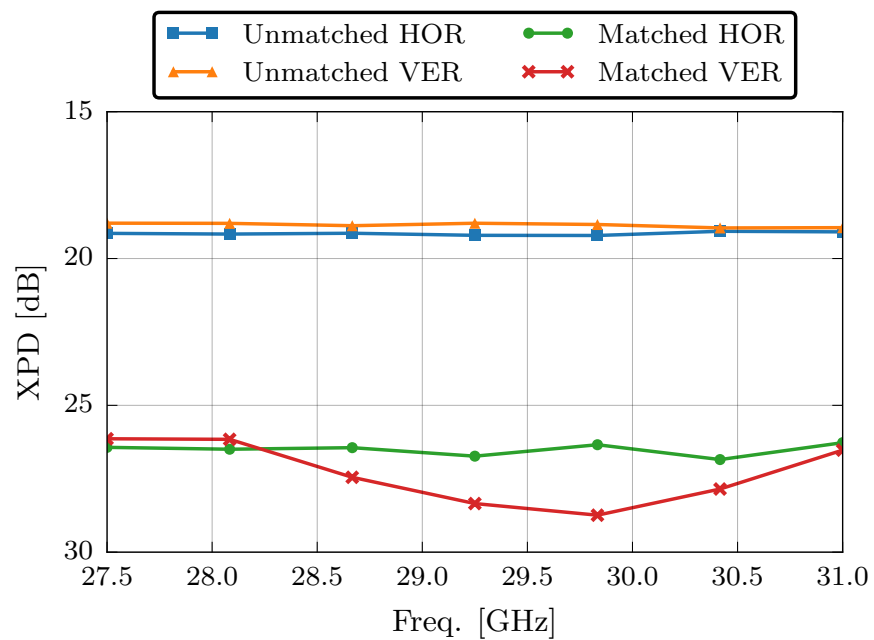

Fig. 3. Cross-polar discrimination (referenced to peak directivity) of the matched feed reflector system compared to unmatched as a function of frequency.

that one might try in order to get a larger improvement and/or larger bandwidth.

\section{CONCLUSION}

An alternative investigation of the waveguide modes required for matched feed operation has been presented. It is confirmed that the addition of the correct $\mathrm{TE}_{21}$ mode to an otherwise low cross polarization feed can provide the required compensation. However, it is also shown that $\mathrm{TE}_{01}$ and $\mathrm{TM}_{01}$ can be used alone or in conjunction with $\mathrm{TE}_{21}$.

A versatile, efficient, and accurate design tool for combined reflector and feed optimization has been presented. This allows generalized scattering matrices computed with BoR-MoM, 3D MoM, or Mode matching to be accurately coupled to a PO or MoM/MLFMM solution of the reflector setup.

Finally, the tool has been used to design a matched feed based on an axially corrugated horn. The feed is modified by putting chips/slots in the corrugations of the horn. An XPD improvement better than $7 \mathrm{~dB}$ is achieved over the $12 \%$ design frequency band.

\section{REFERENCES}

[1] A. W. Rudge and N. A. Adatia, "New class of primary-feed antennas for use with offset parabolic-reflector antennas," Electronics Letters, vol. 11, no. 24 , pp. 597-599, 1975.

2] D. J. Bem, "Electric-field distribution in the focal region of an offset paraboloid," Proceedings of the Institution of Electrical Engineers, vol. 116, no. 5, pp. 679-684, 1969.

[3] P. D. Potter, "A New Horn Antenna with Suppressed Sidelobes and Equal Beamwidths," Microwave Journal, vol. 6, no. 6, pp. 71-78, 1963.

[4] S. B. Sharma, D. A. Pujara, S. B. Chakrabarty, and V. K. Singh, "Improving the Cross-Polar Performance of an Offset Parabolic Reflector Antenna Using a Rectangular Matched Feed," IEEE Antennas and Wireless Propagation Letters, vol. 8, pp. 513-516, 2009.

[5] C. Yang, J. Yu, Y. Yao, X. Liu, and X. Chen, "Novel Corrugated Matched Feed for Cross-Polar Cancellation in Tri-Reflector Compact Range," IEEE Antennas and Wireless Propagation Letters, vol. 13, pp. 1003 1006, 2014. 


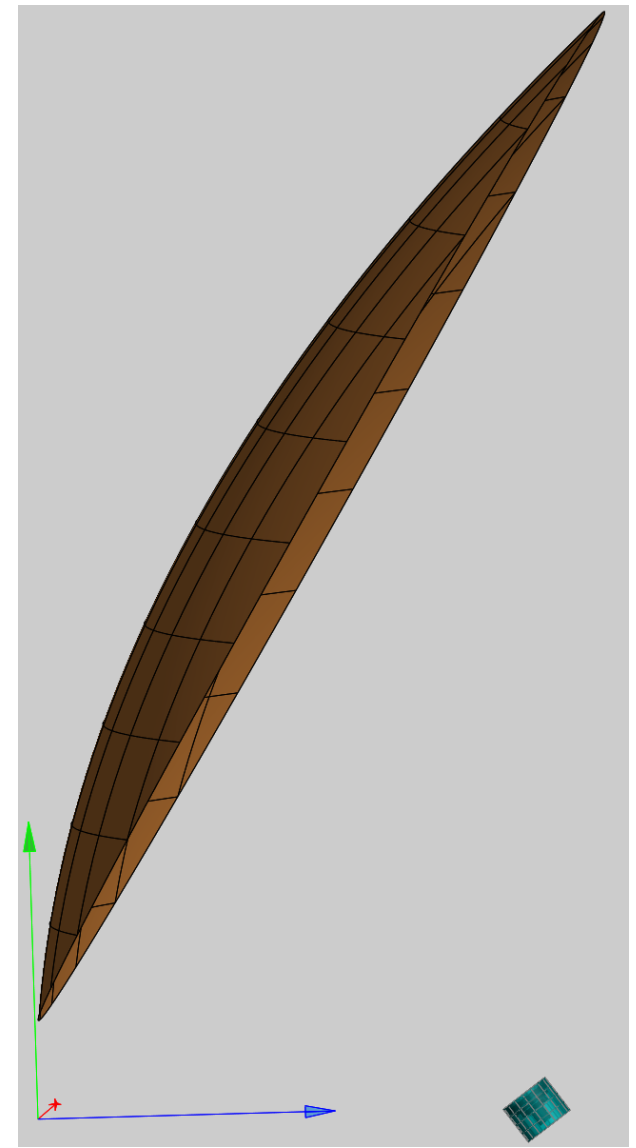

Fig. 4. Model of the entire antenna with $f / D=0.5$.

[6] S. M. Tun and P. J. B. Clarricoats, "Single offset reflector with a matched-feed array," in IEEE Antennas and Propagation Society International Symposium, 1984, pp. 486-489.

[7] Z. A. Pour and L. Shafai, "A Ring Choke Excited Compact DualMode Circular Waveguide Feed for Offset Reflector Antennas," IEEE Transactions on Antennas and Propagation, vol. 60, no. 6, pp. 3011$3015,2012$.

[8] R. Dey, S. B. Chakrabarty, and R. Jyoti, "Broadband Conjugate Matched Feed Horn- A Novel Concept," IEEE Antennas and Wireless Propagation Letters, no. 99, pp. 1-1, 2015 .

[9] A. W. Rudge and N. A. Adatia, "Offset-parabolic-reflector antennas: a review," Proceedings of the IEEE, vol. 66, no. 12, pp. 1592-1618, 1978.

[10] J. R. Risser, "Waveguide and Horn Feeds," in Microwave Antenna Theory and Design, ser. MIT Radiation Laboratory Series. McGrawHill, 1949, no. 12.

[11] "GRASP Software," TICRA, Copenhagen, Denmark, www.ticra.com

[12] E. Jørgensen, P. Meincke, and M. Sabbadini, "Fast and accurate design tool for rotationally symmetric reflector antennas with $3 \mathrm{~d}$ waveguide components and support structures," in Proc. 34th ESA Antenna Workshop, Noordwijk, The Netherlands, 2012.

[13] "CHAMP Software," TICRA, Copenhagen, Denmark, www.ticra.com.

[14] D. Tits, "Low cost high performance antanna for use in transmit/receive satellite terminals." Patent EP1 278 266B1, Sep., 2005.
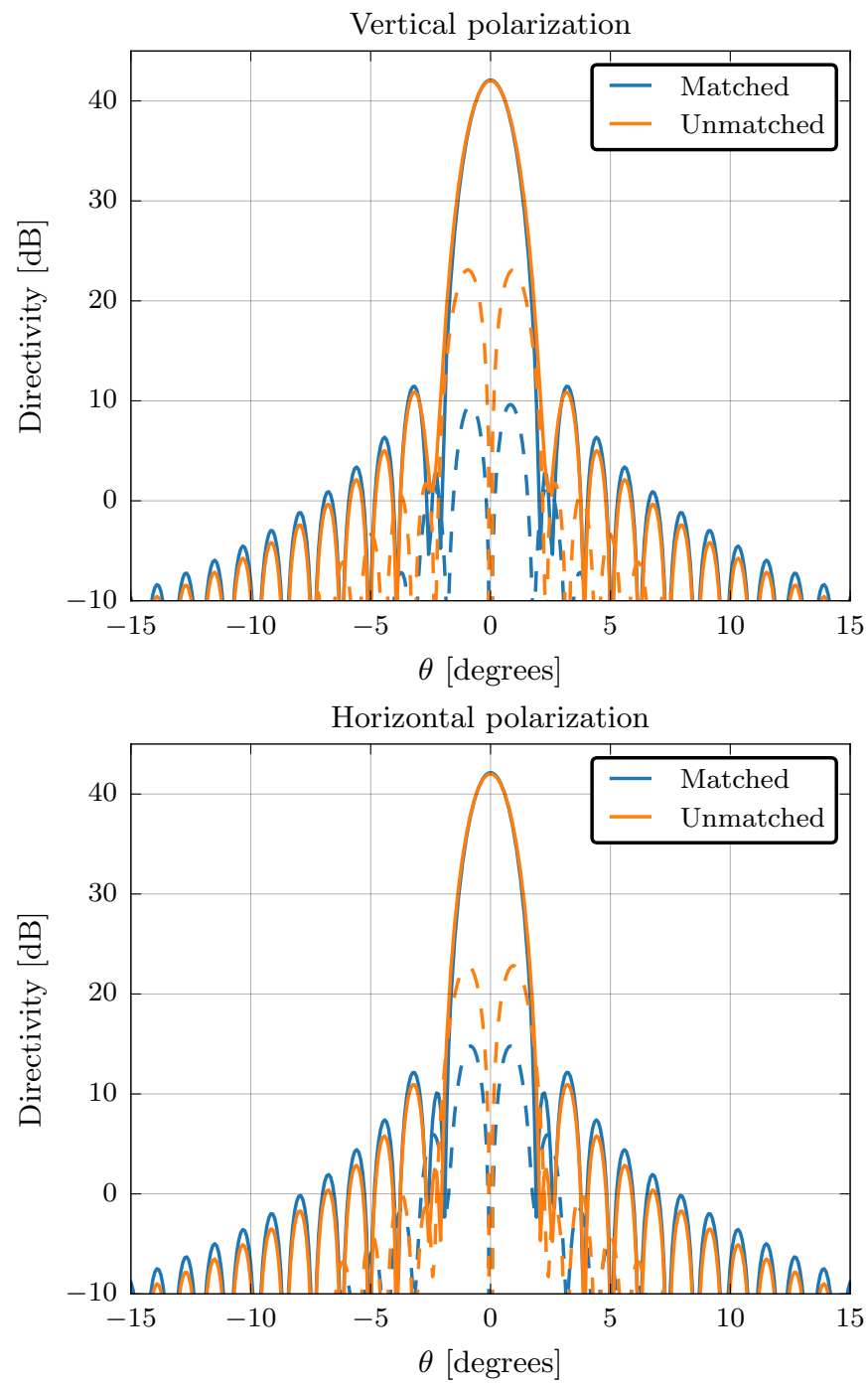

Fig. 5. Pattern cuts in the horizontal plane for the matched and unmatched antennas at $29.25 \mathrm{GHz}$. Solid lines and dashed lines are co- and cross-polar components, respectively. 